\title{
CUIDADO HUMANIZADO Y RIESGOS PSICOSOCIALES: UNA RELACIÓN PERCIBIDA POR PROFESIONALES DE ENFERMERÍA EN CHILE.
}

HUMANIZED CARE AND PSYCHOSOCIAL RISKS: A RELATIONSHIP PERCEIVED BY PROFESSIONAL NURSES IN CHILE

CUIDADOS HUMANIZADOS E RISCOS PSICOSSOCIAIS: UMA ASSOCIAÇÃO PERCEBIDA PELOS PROFISSIONAIS DE ENFERMAGEM NO CHILE

\author{
Sandra Ramos Guajardo \\ Universidad Católica del Maule. Talca, Chile. \\ sramos@ucm.cl \\ ORCID: 0000-0001-8902-9907 \\ Paula Ceballos Vasquez \\ Universidad Católica del Maule. Talca, Chile. \\ ORCID: 0000-0002-3804-5146
}

DOI: https://doi.org/10.22235/ech.v7i1.1537

Recibido: 28/09/2017

Aceptado: 08/03/2018

Resumen: El objetivo de este artículo es evaluar si existe relación entre los factores psicosociales laborales y la entrega de cuidado humanizado por parte enfermeros/as de un hospital público de Chile. El sustento teórico para esta investigación respecto a los factores psicosociales está dado por Karasek y Siegrist. Jean Watson da el sustento disciplinar desde el cuidado humanizado. Se trata de diseño no experimental, transversal, correlacional, con enfoque cuantitativo. Se censó a 240 enfermeros/as que trabajan en un hospital público de Chile. Para la recolección de los datos se utilizó un instrumento dividido en tres partes: Parte I: "Antecedentes personales y laborales", creada por la tesista. Parte II: Cuestionario de Evaluación de riesgos psicosociales en el trabajo SUSESO-ISTAS 21, validado en Chile con un alfa de Cronbach de 0,80. Parte III: Nyberg's Caring Assessment, validado en la población chilena con una consistencia interna de 0,82 . El análisis estadístico fue de tipo descriptivo y correlacional, utilizando Chi cuadrado $(p \leq 0.05)$. Durante la investigación se respetaron los principios éticos de E. Emanuel. Dentro de los resultados se destaca que el $51.67 \%$ de los enfermeros/as reporta una alta percepción de entrega de cuidado humanizado. En las dimensiones de riesgos psicosociales, en tres de ellas presentan un riesgo alto, de nivel 1: Exigencia psicológica, Apoyo social en la empresa y Calidad de liderazgo y doble presencia. Además se encontró una asociación negativa entre la percepción de exposición a riesgos psicosociales y percepción de entrega de cuidado humanizado. Se concluye que la percepción de cuidado humanizado se asocia de forma significativa con la percepción de riesgos psicosociales en el desempeño laboral de enfermeras. 
Palabras clave: Humanización de la Atención; Riesgos Laborales; Atención de enfermería; Enfermería.

\begin{abstract}
The aim of this article is to evaluate if there is relationship between work-related psychosocial factors and the humanized care given by the nurses in a public hospital in Chile. Karasek and Siegrist gave the theoretical support for this research in terms of the psychosocial factors. Jean Watson provides the disciplinary support from humanized caring. The design of the study is not experimental, cross-sectional, with a quantitative approach. A census was performed to 240 nurses that work in a public hospital in Chile. In order to recollect the data, an instrument divided into three parts was used. Part I: "Personal and work-related background", created by the master's degree student. Part II: "Questionnaire of evaluation of work-related psychosocial risks SUSESO-ISTAS 21", validated in Chile with an alpha of Cronbach: 0.80. Part III: Nyberg's Caring Assessment, validated in the Chilean population with an internal consistency of 0.82 . The statistical analysis was descriptive and correlational, using Chi square $(\mathrm{p} \leq 0.05)$. During the present research, the ethical principles of E. Emanuel were followed. The results show that 56.25\% of the nurses report a high perception of humanized care delivery. In terms of psychosocial risks, a high risk is present in three different dimensions (Psychological demand; Social support in the company and Leadership quality and double presence). Besides, a negative association between the exposition to psychosocial risks and the perception of humanized care was found. In conclusion, the perception of humanized care is associated in a relevant way with the perception of psychosocial risks in the working performance of nurses.
\end{abstract}

Key words: Humanization of the attention; work-related risks; Nursing Care; Nursing

Resumo: $O$ objetivo deste artigo é avaliar a existência de relação entre os fatores psicossociais no trabalho e a entrega de cuidado humanizado por parte de enfermeiros/as de um Hospital Público do Chile. O embasamento teórico para esta pesquisa com respeito aos fatores psicossociais está dado por Karasek e Siegrist, e Jean Watson entrega o embasamento disciplinar a partir do cuidado humanizado. É un desenho não experimental, transversal, correlacional, de abordagem quantitativa. Realizou-se um censo aos enfermeiros/as que trabalham em um Hospital Público do Chile. Para a obtenção dos dados, utilizou-se um instrumento dividido em três partes: Parte I: "Antecedentes pessoais e de trabalho", criado por uma tesista, Parte II: Questionário de Avaliação de riscos psicossociais no trabalho SUSESO-ISTAS 21, validado no Chile com um Cronbach alfa: 0,80. Parte III: Nyberg's Caring Assessment, validado na população chilena com consistência interna de 0,82 . A análise estatística foi descritiva e correlacional, usando o quadrado Qui $(\mathrm{p} \leq 0,05)$. Durante a presente pesquisa foram respeitados os princípios éticos de E. Emanuel. Destaca-se que 51.67\% dos enfermeiros (as) referiram uma alta percepção de entrega do cuidado humanizado. Nas dimensões de riscos psicosociais, três apresentam um risco alto (Exigência psicológica; Apoio social na empresa e qualidade de liderança e Dupla presença), portanto enfermeiros (as) dentro de uma organização apresentam um risco alto nivel 1. Além disso, foi encontrada uma associação negativa entre a percepção de 
exposição a riscos psicosociais e percepção da entrega de cuidado humanizado. Conclusões: a percepção do cuidado humanizado está associada de forma significativa com a percepção de riscos psicosociais de desempenho no trabalho de enfermeiras (os).

Palavras-chave: Humanização da Atenção; Riscos no Trabalho; Atenção da enfermagem; Enfermagem.

\section{INTRODUCCIÓN}

La Enfermería es la ciencia y el arte de cuidar de la salud del individuo, familia y comunidad. Dentro de su campo de acción están la promoción y el mantenimiento de la salud, la prevención de enfermedades y la participación en su tratamiento, incluyendo la rehabilitación de la persona, independientemente de la etapa de crecimiento y desarrollo en que se encuentra (1). Expertas indican que uno de los principales objetivos de la Enfermería es mantener al máximo el bienestar físico, mental, social y espiritual del ser humano a través del cuidado, el cual es considerado la esencia de la profesión, y está construido por las acciones transpersonales e intersubjetivas para proteger, mejorar y preservar la humanidad (2). Sin embargo, este cuidado, al parecer, se ha ido deshumanizando día a día. No es extraño observar en la práctica diaria cómo se reconocen a los pacientes por sus números de camas o patologías, no se toman en cuentan las necesidades referidas por ellos/as, entre otros aspectos. Una de las principales razones de este contexto es que aún en el sistema de salud se tiene una mirada biomédica, donde el objetivo es curar la patología, dejando de lado al paciente con su entorno social, familiar y emocional (3).

Reflexionando sobre el tema se descubren muchas situaciones de deshumanización; lo importante es identificar las causas relacionadas con estas conductas con la finalidad de intervenir y mejorar el cuidado entregado. Uno de estos motivos pueden ser los factores psicosociales laborales presentes en las instituciones sanitarias, los cuales al percibirse como negativos pueden afectar la salud del trabajador/a, transformándose en riesgos psicosociales (4). Por otra parte, estos riesgos podrían afectar de alguna manera la calidad del trabajo ejecutado, y por ende, al cuidado que entregan los enfermeros y enfermeras.

En Chile y en el mundo los riesgos psicosociales han generado un aumento de los problemas de salud mental de los trabajadores del sector de servicios, en este caso, trabajadores sanitarios. Estos riesgos tienen relación con nuevas formas de organización de la producción, relaciones laborales, la revolución informática y la introducción masiva de nuevas tecnologías, que se han traducido en diversas formas de intensificación del trabajo, excesiva demanda física y mental, debilitamiento de los colectivos laborales y erosión de las fronteras entre trabajo y vida privada. Estas nuevas formas de organizar el trabajo demandan y tensionan al trabajador emocional y cognitivamente, lo que se traduce en un aumento de la carga psíquica y mental en el trabajo (5). La revisión de literatura permite afirmar que existen varios teóricos que sustentan estudios en relación a riesgos psicosociales, pero su relación con la percepción de cuidado ha sido estudiada más bien a nivel cualitativo. Los modelos teóricos que se explican a continuación son los que sustentan mayor cantidad de investigación y formas de evaluación en el tema. La primera es la propuesta de Karasek y Theorell, la cual vincula tres conceptos, "demanda-control-apoyo 
social" (6-7). Las demandas psicológicas son las exigencias psicológicas que el trabajo implica para la persona. Básicamente hacen referencia a cuánto se trabaja: cantidad o volumen de trabajo, presión de tiempo, nivel de atención, interrupciones imprevistas; por lo tanto, no se circunscriben al trabajo intelectual sino a cualquier tipo de tarea. El modelo predice, en primer lugar, riesgo de enfermedad relacionado con estrés; en segundo lugar, predice relación con comportamiento activo/pasivo. Estos dos mecanismos psicológicos principales, el de tensión psicológica y el de aprendizaje, son independientes, lo que constituye uno de los rasgos esenciales del modelo: su estructura bidimensional (8).

La segunda teoría fue propuesta por Siegrist, quien desarrolló el modelo "Desbalance esfuerzo-recompensa"; en éste se afirma que el rol del trabajo en la adultez es vincular funciones autorreguladoras, como son la autoestima y autoeficacia con la estructura de oportunidades sociales (9). Esto significa que la categoría profesional u ocupacional del trabajador es asociada a una opción de retribución y realización; ser recompensado o apreciado, y de pertenencia significativa a algún grupo. Por lo tanto, en el ambiente laboral las exigencias están presentes continuamente y son capaces de generar sentimientos de ansiedad, miedo, tensión o amenaza que surgen durante el ejercicio de las actividades y requieren respuestas adaptativas, las cuales no siempre son las adecuadas y se vuelven un riesgo laboral (10).

Al desempeñar el cuidado se establece una relación enfermera-usuario, generando afecto, preocupación y responsabilidades de ambos. Este cuidado descubre lo humano, hace aflorar valores, principios, por lo que puede decirse que el cuidado profesional es sinónimo del cuidado humanizado (11). El cuidado humanizado ha sido ampliamente desarrollado por la teórica de Enfermería Jean Watson, quien reconoce que el cuidar es parte fundamental del ser y es el acto más primitivo que un ser humano realiza para efectivamente llegar a ser; este ser es un ser en relación a otro que lo invoca (12). Este llamado desde el otro, absolutamente otro, es la base fundadora del cuidado. Este cuidado es estructurado, formalizado y destinado a satisfacer las necesidades del ser humano con el propósito de promover, mantener o recuperar la salud (13). Watson postula que ante el riesgo de deshumanización en el cuidado del paciente a causa de la gran restructuración administrativa de la mayoría de los sistemas de cuidado de salud en el mundo, se hace necesario el rescate del aspecto humano, espiritual y transpersonal en la práctica clínica, administrativa, educativa y de investigación por parte de los profesionales en el campo de la enfermería. Es por lo expuesto que la experta hace un llamado a mejorar la enfermería actual y entrega un marco disciplinario con el cual trabajar en nuestra práctica diaria en los diferentes ámbitos de la enfermería, visualizando a la persona desde una mirada transformadora e integral, alejándose del modelo biomédico (2).

Así, la entrega de un cuidado humanizado sería prioritario para Enfermería, ya que es una profesión en la que se debe partir de un autoconocimiento; además profesionalmente se requiere de un dominio y continua reflexión sobre problemas de interacción humana desde un punto de vista ético, social y político. Al respecto, autores indican que la sociedad requiere de este tipo de cuidado, basado en una relación humana entre los diversos participantes en el acto de cuidar (14). Sin embargo este contexto, si bien positivo para el usuario, puede generar en los profesionales riesgos laborales. Los enfermeros y enfermeras 
deben saber que al trabajar con seres humanos, sumado a los factores laborales presentes en los ambientes de trabajo, se puede ver afectada su salud, ya sea física, mental o social, y eso puede afectar la labor de cuidar. Por consecuencia, esta entrega de cuidados va a ser de menor calidad, y no solo en lo procedimental, sino también en el trato con el otro, que se ve reflejado en su deshumanización. Por lo tanto el enunciado del problema expuesto responde a que se desconoce el impacto que los riesgos psicosociales laborales pueden tener en la percepción de entrega de cuidados humanizado por parte de los/as profesionales de enfermería de un hospital público de Chile. Así, este estudio emerge con el objetivo de evaluar si existe relación entre los riesgos psicosociales en el trabajo y la entrega de cuidado humanizado por parte de los enfermeros/as de ese hospital.

\section{METODOLOGÍA}

El diseño de la investigación fue no experimental, transversal, correlacional con enfoque cuantitativo (15). Se destaca que en todas las etapas de esta investigación se respetaron los principios éticos de Emanuel (16). Los datos fueron tomados en un hospital público de la Región del Maule de Chile entre noviembre 2016 y enero 2017. La población de estudio se logró por medio de un censo efectivo, ya que de una población total de 325 enfermeros/as, se obtuvo una población diana de 258 trabajadores/as (ya que cumplían con los criterios de inclusión establecidos para esta investigación). De éstos, 240 fueron censados, por lo que la población de estudio corresponde al $93 \%$ del total de la población diana. Los criterios de inclusión fueron: ser enfermera o enfermero titulado, que estuviesen trabajando formalmente (contrata, reemplazo, indefinido), desempeñando funciones asistenciales prestando cuidados directos a usuarios/as y que firmaran el consentimiento informado, autorizando al investigador. Y los criterios de exclusión: desempeñar labores mayoritariamente administrativas o de gestión, encontrarse con vacaciones, licencia o permiso cuando se recolectaran los datos y desempeñar labores con pacientes con trastornos mentales graves.

La recolección de datos se llevó a cabo luego de la aprobación del Comité de Ética-Científico de la Universidad Católica del Maule y del Servicio de Salud del Maule. Se comenzó con la lectura y entrega del consentimiento informado, el cual debió ser firmado, entregándose una copia al participante. Luego se informó el tiempo que le tomaría la aplicación de los instrumentos, y se procedió a contestarlos. La aplicación fue realizada por la investigadora principal, en caso de que existieran consultas o dudas en relación a los instrumentos. El instrumento utilizado para la recolección de datos estuvo conformado por tres partes. Parte I (Instrumento A): "Antecedentes personales y laborales", creado por la investigadora, con el cual se recolectaron las variables biosociodemográficas y datos laborales. Parte II (Instrumento B): "Cuestionario de Evaluación de riesgos psicosociales en el trabajo SUSESO-ISTAS 21”. Este instrumento resultó de la adaptación, validación y estandarización del Método ISTAS 21-COPSOQ en la población trabajadora chilena. Este procedimiento fue realizado por la Escuela de Salud Pública de la Universidad de Chile en 2008, obteniendo un Coeficiente alfa de Cronbach mayor a 0,80, evidenciando una adecuada consistencia interna (17). Parte III (Instrumento C): Nyberg's Caring Assessment 
(NCA), desarrollado en el año 1990 por Jan Nyberg durante su estudio doctoral en la Universidad de Colorado, pero la versión que se utilizó en el presente estudio es la versión traducida y validada por la Doctora en Enfermería Margarita Poblete, con población chilena, con un Coeficiente alfa de Cronbach: 0,82 (18).

Los datos fueron procesados y analizados utilizando el programa SPSS versión 18. Se realizó análisis exploratorio de los datos mediante estadísticos descriptivos, por medio de tablas de frecuencia, medidas de tendencia central y de variabilidad. Luego se realizó análisis estadístico con un $95 \%$ de confianza, para lo cual se utilizó estadígrafos no paramétricos (Chi-cuadrado y árbol de clasificación).

\section{RESULTADOS}

En las variables personales, se puede observar que en relación a la edad, los enfermeros tienen una edad promedio de 31.46 años, siendo el rango de mayor representatividad el de 23 a 30 años, correspondiente a un $60 \%$. En relación al sexo de los participantes, el $82.9 \%$ correspondía al sexo femenino. De los participantes de la población de estudio un $67.1 \%$ reportó tener pareja, y respecto a la variable hijos, un $62.9 \%$ no tiene hijos. Por otra parte, de las variables laborales destaca que en promedio los trabajadores participantes reportan una media de 4.9 años de antigüedad en el puesto de trabajo. Específicamente, un 56,7\% explicita una antigüedad entre 1 y 5 años, seguido de 6 a 10 años con un $17.5 \%$ y con menos de un año el $15.8 \%$. En relación al grado de contratación, un alto porcentaje se encuentra en grado 15 , correspondiendo a un $45.8 \%$, seguido de grado 14 con un $24.6 \%$. En la situación contractual con la empresa la mayoría está contratado a plazo fijo (contrata) correspondiendo al $85.8 \%$. En relación a la jornada el $73.8 \%$ trabaja en cuarto turno, además el $87.1 \%$ solo trabaja para el hospital y el $17.5 \%$ tiene estudios posteriores.

En la tabla $\mathrm{N}^{\circ} 1$ de niveles de riesgo psicosocial se puede observar que en la dimensión Exigencias psicológicas el $57.1 \%$ percibe una alta exposición a riesgo. En la dimensión Trabajo activo y desarrollo de habilidades, el $46.7 \%$ percibe riesgo medio. En la dimensión Apoyo social en la empresa y calidad de liderazgo el $47.5 \%$ percibe alta exposición a riesgo. En la dimensión Compensaciones el $43.3 \%$ percibe riesgo medio. En la dimensión Doble presencia el $55 \%$ percibe riesgo alto. 
Tabla 1: Niveles de riesgo psicosocial percibido por las enfermeras /os participantes del estudio

$\$$

\begin{tabular}{l|l|l}
\hline Riesgos psicosociales por dimensiones & $\mathrm{f}$ & $\%$ \\
\hline $\begin{array}{l}\text { Dimensión Exigencias psicológicas } \\
\text { Promedio: 12.09, Desviación típica: 2.44, Rango: 7-19 }\end{array}$ \\
\hline Bajo (0-8) & 19 & 7.9 \\
\hline Medio (9-11) & 84 & 35 \\
\hline Alto (12-20) & 137 & 57.1 \\
\hline $\mathrm{n}$ & 240 & 100 \\
\hline
\end{tabular}

Dimensión trabajo activo y desarrollo de habilidades

Promedio: 6.56, Desviación típica: 2.7, Rango: 2-13

\begin{tabular}{l|l|l}
\hline Bajo (0-5) & 80 & 33.3 \\
\hline Medio (6-8) & 112 & 46.7 \\
\hline Alto (9-20) & 48 & 20 \\
\hline $\mathrm{n}$ & 240 & 100 \\
\hline
\end{tabular}

Dimensión apoyo social en la empresa y calidad de liderazgo

Promedio: 6.3, Desviación típica: 2.7, Rango: 0-14

\begin{tabular}{l|l|l}
\hline Bajo (0-3) & 37 & 15.4 \\
\hline Medio (4-6) & 89 & 37.1 \\
\hline Alto (7-20) & 11 & 47.5 \\
\hline $\mathrm{n}$ & 240 & 100 \\
\hline
\end{tabular}

Dimensión compensaciones

Promedio: 4.4, Desviación típica: 2.52, Rango: 0-12

\begin{tabular}{l|l|l}
\hline Bajo (0-2) & 59 & 24.6 \\
\hline Medio (3-5) & 104 & 43.3 \\
\hline Alto (6-12) & 77 & 32.1 \\
\hline $\mathrm{n}$ & 240 & 100 \\
\hline
\end{tabular}

Dimensión doble presencia

Promedio: 3.8 , Desviación típica: 1.9 , Rango: $0-8$

\begin{tabular}{l|l|l}
\hline Bajo (0-1) & 27 & 11.3 \\
\hline Medio (2-3) & 81 & 33.8 \\
\hline Alto (4-8) & 132 & 55 \\
\hline $\mathrm{n}$ & 240 & 100 \\
\hline
\end{tabular}

Fuente: Elaboración propia (2017) 
En gráfico $\mathrm{N}^{\circ} 1$, se puede observar que en relación a la percepción de entrega de cuidado, que los participantes reportan una alta entrega de cuidados humanizados $(51.67 \%)$.

Gráfico $\mathbf{N}^{\circ}$ 1: Cuidado humanizado percibido por las enfermeras /os participantes del estudio

Percepción de cuidado humanizado

$\square$ Menor percepción

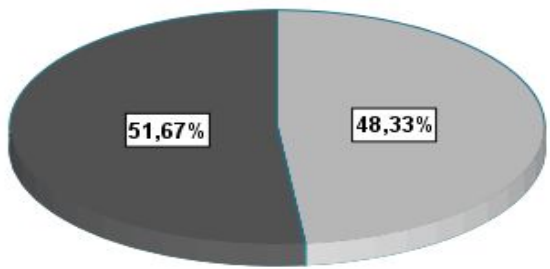

Fuente: Elaboración propia (2017)

En el gráfico $\mathrm{N}^{\circ}$ 2, se puede ver que la asociación de la dimensión Trabajo activo y desarrollo de habilidades y percepción de cuidado humanizado es significativa $(p=0.033)$, y se observa que al percibir niveles de exposición a riesgos psicosociales bajos en esta categoría reportan una alta percepción de entrega de cuidado humanizado.

Gráfico $\mathbf{N}^{\circ}$ 2: Asociación entre la dimensión Trabajo activo y Desarrollo de habilidades y el cuidado humanizado percibido por las enfermeras /os participantes del estudio
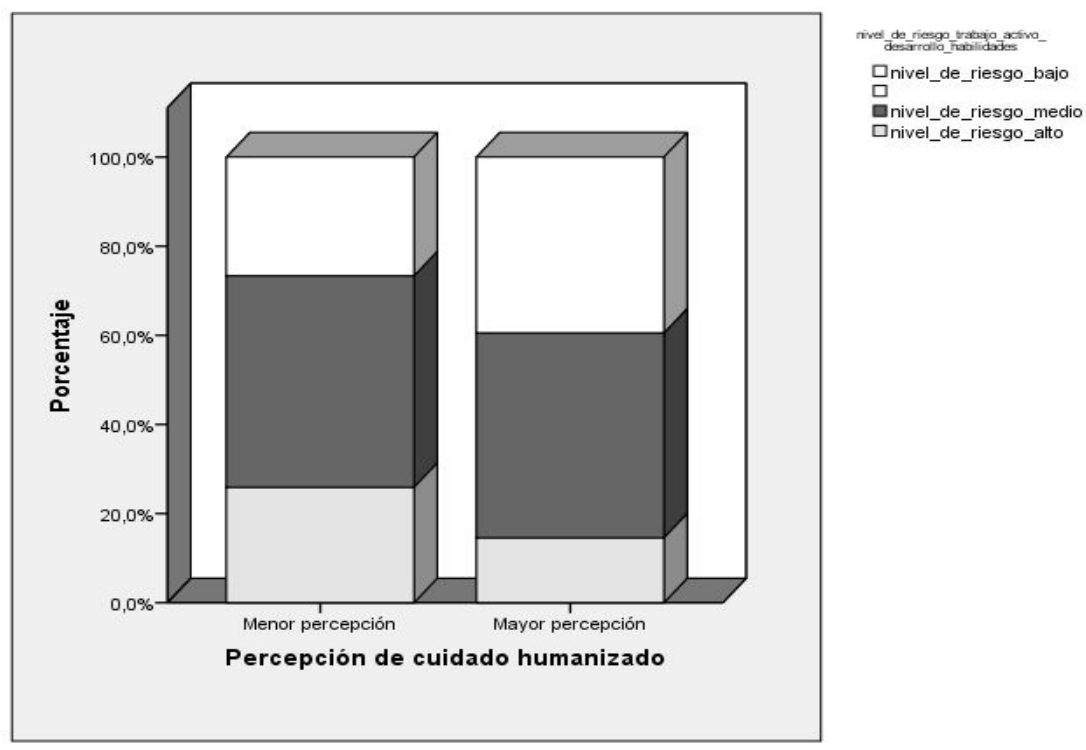

Fuente: Elaboración propia (2017) 
En el gráfico $\mathrm{N}^{\circ} 3$ se puede ver que la asociación de la dimensión Apoyo social y calidad de liderazgo y percepción de cuidado humanizado es significativa $(p=0.035)$, observándose además que los participantes de este estudio, al percibir niveles de exposición a riesgos psicosociales más altos en esta categoría, reportan una baja percepción en entrega de cuidado humanizado.

Gráfico N 3: Asociación entre dimensión Apoyo social en la empresa y Calidad de liderazgo y el cuidado humanizado percibido por las enfermeras /os participantes del estudio
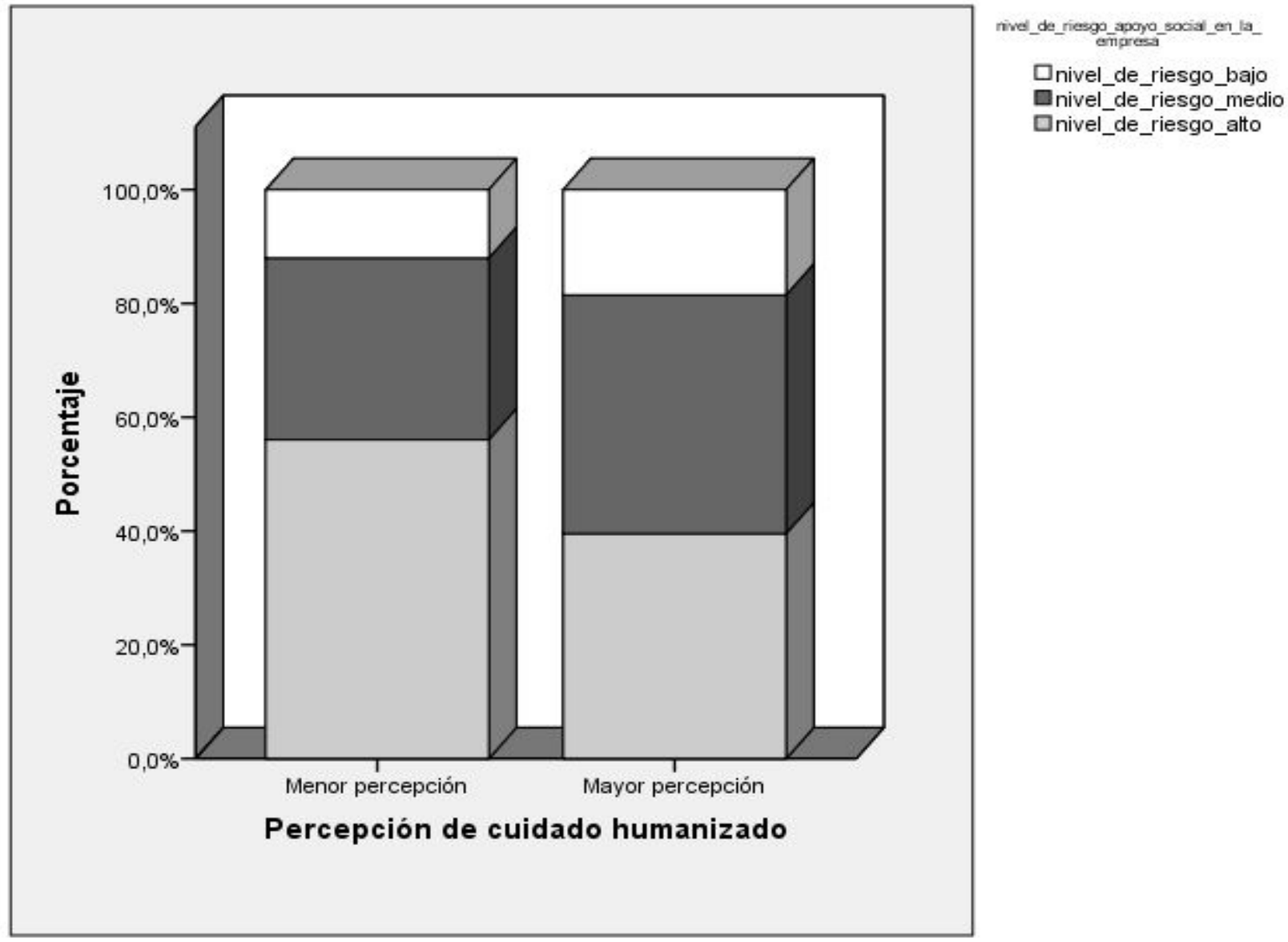

Fuente: Elaboración propia (2017)

En el gráfico $\mathrm{N}^{\circ} 4$ se puede ver que la asociación de la dimensión Doble presencia con cuidado humanizado es significativo $(p=0.015)$. Este resultado indica que los participantes del estudio, al percibir menor riesgo psicosocial es esta categoría, perciben alta entrega de cuidado humanizado. 
Gráfico $\mathbf{N}^{\circ}$ 4: Asociación entre la dimensión Doble presencia y el cuidado humanizado percibido por las enfermeras /os participantes del estudio.

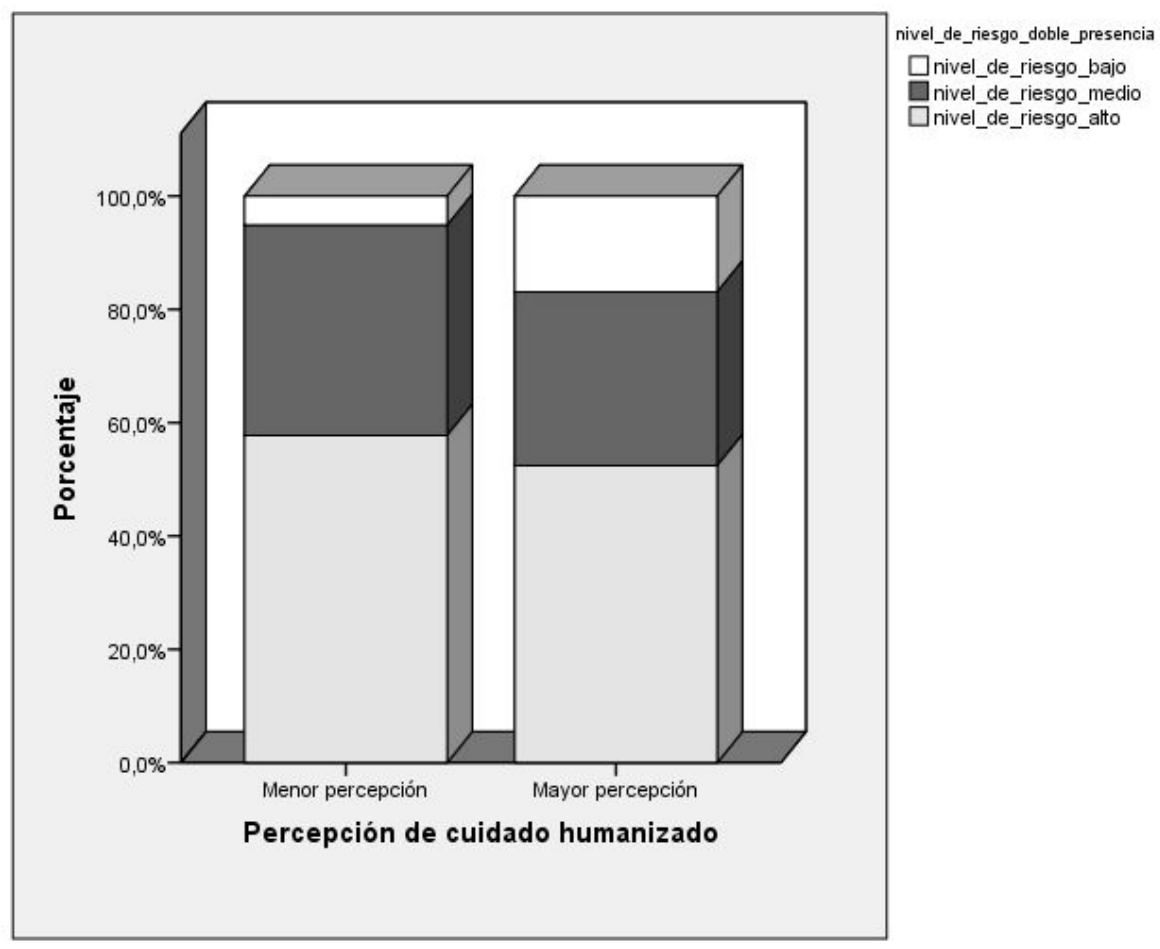

Fuente: Elaboración propia (2017)

\section{DISCUSIÓN}

Si se analizan los resultados de los niveles de exposición a riesgos psicosociales desde el modelo Demanda-Control-Apoyo social de los enfermeros y enfermeras participantes, éstos dan cuenta de un trabajo de alta demanda, ya que reportan una alta exposición a riesgos en la dimensión Exigencias psicológicas más una baja percepción de control sobre lo que hacen (7). Sumado a lo señalado, perciben riesgo medio en la dimensión trabajo activo y desarrollo de habilidades; se percibe un trabajo de alto nivel de estrés (según la teoría ya señalada). Expertos indican que el amortiguador que permite sobrellevar un trabajo de alto estrés es el apoyo social, pero en el presente estudio la percepción de riesgo en esta dimensión también es alta, por lo que se vuelve un trabajo de alto riesgo, lo que puede generar problemas graves a la salud de este grupo de trabajadores. Similar es el resultado de otro estudio, en donde los profesionales percibieron bajo control; creían no poseer las capacidades para ejecutar ciertos cursos de acción para manejar situación en su trabajo y se mostraron más susceptibles a afecciones de salud mental (19). Por otra parte, los resultados de este estudio son opuestos a los encontrados por Díaz y Mauro, en donde los profesionales de la salud reportaron alta demanda, pero a la vez, alto control y alto soporte social (5). 
Si se analizan los resultados bajo la perspectiva del modelo esfuerzo-recompensa, se observa que los enfermeros y enfermeras estudiadas perciben mayormente un riesgo medio en la dimensión Compensaciones y riesgo alto en Exigencias psicológicas, por lo que se puede generar un desbalance en la motivación del trabajador/a, exponiéndolos a angustia emocional, y por ende, a trastornos físicos o psíquicos asociados a su trabajo (9).

En relación a las dimensiones de riesgos psicosociales se observa que en las dimensiones Exigencia psicológica y Doble presencia los trabajadores participantes perciben una alta exposición a riesgos, y en las dimensiones Trabajo activo y desarrollo de habilidades y Compensaciones perciben un nivel medio de exposición a riesgos. Estos resultados son similares a los encontrados por Ceballos et. al., en donde también aplicaron cuestionario SUSESO ISTAS-21 en una población de enfermeros (as) de unidades críticas de zona centro sur de Chile (20). Contradice los hallazgos de Moncada et. al., en donde se reportaron bajas puntuaciones en las dimensiones de doble presencia y emociones, en donde los participantes eran una población asalariada de diferentes áreas (21). Esta contradicción se puede explicar debido al tipo de trabajadores; en esta investigación, y en el estudio de Ceballos et. al. la población estudiada fueron enfermeras, y en el estudio de Moncada participaron profesionales de todas las áreas (20-21). Se destaca que el trabajo diario de enfermeras/os requiere estar en contacto con un tercero, siendo una labor de servicio con altas responsabilidades asociadas, sumado a que la mayoría de los trabajadores participantes son de sexo femenino, por lo que cumplen una doble función social (Trabajadora/dueña de casa).

Los resultados obtenidos en la percepción de cuidado humanizado indican que un alto porcentaje de enfermeros y enfermeras perciben una alta entrega de cuidado humanizado, a pesar de encontrarse con un nivel alto de percepción de riesgo psicosocial. Este resultado se puede sustentar desde la teoría de Watson (2), quien señala que el cuidado es un tipo especial de relación que trasciende el tiempo y el espacio y continúa como parte del mayor complejo patrón de vida de la enfermera y del usuario/a. Si se analizan estos resultados obtenidos se observa que a pesar de las dificultades del trabajo, los enfermeros y enfermeras perciben otorgar un cuidado de acuerdo a los procesos caritas planteados por Watson. Empíricamente, los resultados de este estudio son similares a los reportados por otros autores, en donde el cuidado humanizado se ve reflejado en el acto de cuidar, servir y tratar a los pacientes con afecto, pero también destacan que la satisfacción laboral es un punto importante para lograr un adecuado cuidado (22). A pesar de encontrarse más del $50 \%$ con una percepción alta de cuidado humanizado, no es menor el porcentaje que presenta un baja percepción: el $43.75 \%$. Este resultado es similar al obtenido en otras investigaciones, en donde se hace hincapié en que las enfermeras (os) tienen un significado teórico claro del cuidado, pero al llevarlo a la práctica éste se dificulta debido a diferentes factores, como por ejemplo la organización de la institución, rutinas hospitalarias, entre otros que se podrían asociar a la percepción de los factores de riesgos psicosociales (23-25).

En relación a los resultados obtenidos al buscar una asociación entre la percepción de entrega de cuidado humanizado y los riesgos psicosociales se encuentra que las 
dimensiones Trabajo activo y desarrollo de habilidades, Apoyo social en la empresa y calidad de liderazgo, Compensaciones y Doble presencia presentan una asociación inversamente significativa con la percepción de cuidado humanizado reportada por los trabajadores participantes de este estudio. De esta forma, el profesional de Enfermería al percibir alta exposición a riesgos psicosociales, su percepción de entrega de cuidado humanizado baja. Estos resultados son similares a otras investigaciones donde se analizaron las condiciones laborales de los enfermeros y enfermeras, logrando concluir que el déficit de recurso humano, sobrecarga laboral, escasa capacitación, baja retribución económica, y por ende, condiciones laborales precarias, fueron identificados como obstáculos para ejercer un cuidado de calidad (26-28). Por lo tanto, contar con ambientes laborales saludables debería impactar el cuidado de manera positiva. Teniendo en cuenta esto, como estamento de enfermería se debería enfrentar la problemática laboral y luchar por conseguir ambientes laborales más saludables, ya que tendrían un impacto final en la calidad de los cuidados entregados a los usuarios, lo que mejoraría la salud pública de nuestro país.

\section{CONCLUSIONES}

Se puede concluir que los riesgos psicosociales tienen relación con la percepción de cuidado humanizado, encontrando asociación significativa en tres dimensiones. Así, a mayor percepción de riesgo psicosocial, menor entrega de cuidado humanizado. $A$ nivel profesional, las enfermeras y enfermeros deben enfrentar las problemáticas respecto a los ambientes laborales, luchando por ambientes más saludables de trabajo, evitando así la exposición altas a riesgos psicosociales y mejorando por ende la calidad de los cuidados que entregan.

\section{AGRADECIMIENTOS}

Este trabajo fue financiado por el Proyecto Interno línea de tesis de postgrado UCM 435106 dependiente de la Vicerrectoría de Investigación y Postgrado de la Universidad Católica del Maule. Parte de este artículo es derivado de la Tesis de Magíster denominada Percepción del Cuidado humanizado entregado por los profesionales de Enfermería en relación a los factores psicosociales, del Programa de Magíster en Enfermería de la Universidad Católica del Maule, Chile.

\section{REFERENCIAS BIBLIOGRÁFICAS}

1) CPE Puerto Rico. Reglamento de la junta examinadora de enfermeras y enfermeros de puerto rico para la implantación de la ley número 9, 1984. Disponible en: http://cpepr.org/images/PDF/REGLAMENTO\%205069.pdf

2) Watson J. Watson's theory of human caring and subjective living Experiences: carative factors/caritas processes as a Disciplinary guide to the professional nursing practice. Texto Context Enferm. 2007; 16(1): 129-35.

3) Ceballos P. Desde los ámbitos de enfermería, analizando el cuidado humanizado, Ciencia y Enfermería. 2010; 16 (1): 31-5. 
4) Boada J, Ficapal-Cusí. (2012). Salud y trabajo los nuevos y emergentes riesgos psicosociales. Editorial UOC. Barcelona: España; 2012.

5) Díaz X, Mauro A. Riesgos psicosociales y salud mental de trabajadores y trabajadoras del sector salud en Chile. E., Ansoleaga, O. Artaza, \& J. Suarez, (Eds.) Personas que cuidan personas: dimensión humana y trabajo en salud. Santiago, Chile: OPS/OMS. 2012: 146-164. Disponible en: http://scielo.proyectoaraucaria.cl/documentos/20130627103555Riesgos\%20psicoso ciales $\% 20 \mathrm{y} \% 20$ salud $\% 20$ mental $\% 20$ de $\% 20$ trabajadores $\% 20 \mathrm{y} \% 20$ trabajadoras $\% 20$ del $\% 20$ sector $\% 20$ salud $\% 20$ en $\% 20$ Chile.pdf

6) Karasek R. Job demand, job decision latitude, and mental strain: implication for job redesign. Rev Administrative Science Quarterly. 1979; (24).

7) Karasek R, Baker D, Marxer F, Ahlbom A, Theorrel T. Job Decision latitude, job demands, and cardiovascular disease: A prospective study of Swedish men. AJPH. 1981; 71 (7): 694-705.

8) Vega S. Riesgo psicosocial: el modelo demanda-control-apoyo social. Ministerio del trabajo y asuntos sociales España. 2001. Disponible en : http://www.insht.es/InshtWeb/Contenidos/Documentacion/FichasTecnicas/NTP/Fic heros/601a700/ntp_603.pdf .

9) Siegrist J. Adverse health Effects of high-effort/ low-reward conditions. Journal of occupational health psychology. 1996; 1(1): 27-41.

10) Grazziano E, Ferraz E. Impacto del estrés ocupacional y burnout en enfermeros. Revista enfermería global. 2014; 9(1): s/p.

11) Hinostroza N. El cuidado profesional de enfermería un enfoque humanístico, Rev.enferm.vanguard. 2014; 2 (1):86-97.

12) Watson J. Caring science as sacred science. Philadelphia: F.A. Davis Company; 2005.

13) Urra E, Jara A, García M. Algunos aspectos esenciales del pensamiento de Jean Watson y su teoría de cuidados transpersonales. Ciencia y enfermería. 2011; XVIII (3):11-22.

14) González L, Velandia A, Flores V. Humanización del cuidado de enfermería. De la formación a la práctica clínica. Revista CONAMED 2009; 40- 43.

15) Grove S, Gray J, Burns N. Investigación en enfermería. Desarrollo de la práctica enfermera basada en la evidencia. 6 ed. Barcelona: España. Elsevier; 2016. P 209-246.

16) Emanuel E, Wendler D, Grandy C. ¿What make clinical research ethical?. JAMA: 2701-2711.

17) Alvarado R, Pérez J, Saavedra N, Fuentealba C, Alarcón A, Marchetti N, Aranda W. Validación de un cuestionario para evaluar riesgo psicosocial en el ambiente laboral en Chile. Rev Med Chile. 2012; 140: 1154-63.

18) Poblete M, Valenzuela S, Merino J. Validación de dos escalas utilizadas en la medición del cuidado humano transpersonal basadas en la teoría de Jean Watson. Aquichán. 2012; 12(1):8-21. 
19) Juárez A. Factores psicosociales relacionados con la salud mental en profesionales de servicios humanos de México. Revista ciencia y trabajo. 2004; 6(14): 189-96.

20) Ceballos P, Rolo G, Hernández E, Díaz D, Paravic T, Burgos M. Factores psicosociales e carga mental de trabajo: una realidad percibida de los enfermeros en Unidades de Terapia Intensiva. Revista Latino-Americana de Enfermagem. 2015; 23(2), 315-22.

21) Moncada S, Serrano C, Font A, Galtés A, Navarro A. Exposición a riesgos psicosociales entre la población asalariada en España: valores de referencia de las 21 dimensiones del cuestionario COPSQ ISTAS 21. Rev Esp Salud Pública. 2004-2005; 82: 667-75.

22) Afio J, Soares E, Miranda L, Ponte R. Cuidado humanizado en terapia intensiva: un estudio reflexivo. Esc Anna Nery Enferm. 2007; 11(2):325-30.

23) Vila V, Rossi L. Significado cultural del cuidado humanizado en la unidad de terapia intensiva: "Mucho se habla y poco se ve". Rev Latino-am Enfermagem. 2002; 10(2):137-44.

24) Barbosa L, Azevedo S. Dialéctica del cuidado humanizado en la UCI: contradicciones entre el discurso y la práctica profesional del enfermero. Rev Esc Enferm USP. 2007; 42 (1): 66-72.

25) Almeida I, Paes M. Atención humanizada de enfermería: el agir con respeto en un hospital universitario. Revista brasileira de enfermagem REBEn. 2007; 60 (5):546-51.

26) Oliveira S, Queiroz J, Melo R. Cuidando y humanizando: obstáculos que dificultan estas prácticas. Rev. Enferm. UER. 2010; 18 (4): 598-603.

27) Cogollo Z, Gómez E. Condiciones laborales en enfermeras de Cartagena, Colombia. Revista avances en Enfermería. 2010; (1): 31-8.

28) Mesa L, Romero M. (2010). Profesionales de enfermería y cuidado en las condiciones laborales actuales. Investigación en Enfermería: Imagen y Desarrollo. $2010 ; 12(2), 55-92$. 